\title{
Implicações de custo financeiro e recursos humanos em assistência odontológica no Brasil segundo o modelo escandinavo*
}

\section{Implications in terms of costs and manpower of using the Scandinavian dental care model in Brazil}

\author{
Jefferson L. Traebert
}

Centro de Estudos de Odontologia em Saúde Coletiva de Santa Catarina. Florianópolis, SC - Brasil

\begin{abstract}
Resumo
Objetivou-se detectar e analisar as implicações em custos e recursos humanos em odontologia de uma hipotética aplicação dos conceitos escandinavos de atenção odontológica no Brasil. Duas sociedades foram escolhidas para serem estudadas: Suécia, país da Escandinávia, e Santa Catarina, Estado da região Sul do Brasil. A análise teórica dessas implicações foi realizada a partir da determinação das necessidades de recursos humanos em odontologia, considerando-se os altos índices de doenças bucais no Estado brasileiro. Utilizou-se o programa de software "Health Through Oral Health" da Organização Mundial da SaúdeFederação Dentária Internacional (1989). Os resultados mostraram alto custo financeiro e a necessidade de se aumentar em até $85 \%$ o recurso humano disponível para atuar em saúde bucal no Estado de Santa Catarina. Concluiu-se que os princípios de alta provisão de recursos humanos e financeiros, característicos do modelo escandinavo, não serviriam para serem adotados por Santa Catarina e, extensivamente, pelo Brasil.
\end{abstract}

Serviços de saúde bucal, organização \& administração. Odontologia em saúde pública, economia. Recursos humanos em odontologia, provisão \& distribuição.

\begin{abstract}
The implications in terms of dental manpower and costs of applying the Scandinavian system of dental care in Brazil are specified and analysed. Two societies were chosen for study: those of Sweden, in Scandinavia and Santa Catarina, State in southern Brazil. The theoretical analysis of the implications was undertaken on the basis of an estimate of dental and auxiliary manpower required to deal with the high levels of oral disease and needs (caries, teeth needing extraction, periodontal diseases and the need for full dentures) in Santa Catarina. This estimate was undertaken by means of the World Health Organization-Fédération Dentaire Internationale (WHO-FDI) Model "Health
\end{abstract}

* Baseado na dissertação de mestrado entitulada "Implications in manpower and costs of using the Swedish model of dental care in Santa CatarinaBrazil", apresentada à School of Medicine of University College London, Londres, 1991.

Correspondência para/Correspondence to: Jefferson L. Traebert - Rua Cel. Teixeira Oliveira, 288 - 88160-000 Biguaçu, SC - Brasil Recebido em 21.6.1995. Reapresentado em 2.5.1996. Aprovado em 24.5.1996. 
Through Oral Health" (1989). The implications in costs, taking the total Swedish expenditure per dentist as a basis, were calculated and applied to the manpower calles for by the WHO-FDI model. The high costs resulting showed that the application of the Swedish model of dental care to Santa Catarina would be unrealistic. The implications in terms of manpower were calculated by applying the manpower ratios considered desirable in Sweden to Santa Catarina. In order to achieve the Swedish dentist: population ratio, Santa Catarina would need $85 \%$ more dentists that it had in 1990. Regarding auxiliary personel, the Brazilian State of Santa Catarina would need to train a larg number of new personnel in order to attain the Swedish auxiliary: dentist ratio. In the ligth of the findings of this study, the adoption of the Swedish model of providing dental care by Santa Catarina in terms of costs and manpower is not feasible. Therefore, alternative approaches for the improvement of oral health in Santa Catarina and in the rest to Brazil should be sought. These approaches should take consider political, cultural and socio-economic aspects of Brazilian society into consideration.

Dental health services, organization and administration. Public health dentistry, economy. Dental staff, supply and distribution.

\section{INTRODUÇÃO}

A colaboração científica internacional tem sido estimulada em todas as áreas do conhecimento. Na odontologia isto não é diferente. São provas incontestes os estudos colaborativos internacionais, patrocinados pela Organização Mundial de Saúde ${ }^{31}$, em 1974 e 1988, visando a buscar informações relacionadas às várias modalidades de atenção à saúde bucal.

Evang $^{6}$ (1975) justifica a troca de experiências internacionais apoiando-se no fato de que áreas específicas dos serviços de saúde não se diferenciam significativamente de um lugar para outro. Como exemplos, cita equipamentos e padrão de construção, conhecimento médico em diagnóstico, tratamento e reabilitação. $\mathrm{O}$ autor esclarece ainda que os serviços de saúde podem ser caracterizados como um setor flexível da sociedade, o que permite adaptações.

Barmes $^{1}$ (1975) mostrou que, de uma forma geral, as principais características dos possíveis modelos assistenciais são: formas de pagamento (direto, indireto), tipo de serviço prestado (curativo, preventivo), população alvo (demanda, necessidade), mãode-obra empregada (privada, pública) e tipo de recursos humanos (profissionais, auxiliares).

Em contrapartida, é reconhecido que, embora as trocas de experiências internacionais sejam úteis, o modelo de atenção à saúde bucal em qualquer país é fração bastante pequena de seu sistema de bem-estar social. Os serviços de saúde são parte integrante da estrutura política, econômica e administrativa de qualquer sociedade, e só podem ser entendidos nes- te contexto ${ }^{6}$. A simples importação de um modelo assistencial de uma sociedade, sem levar em consideração as diferenças socioeconômicas, políticas e culturais, não tem produzido efeitos benéficos. Navarro $^{20}$ (1974) mostrou os efeitos negativos da adoção de conceitos de cuidados médicos e de serviços de saúde, dos países desenvolvidos, na América Latina.

Embora haja até um certo consenso sobre este assunto, diversos países do Terceiro Mundo insistem em adotar princípios de atenção à saúde utilizados em países desenvolvidos. Medidas de orientação curativista, baseadas em atendimento hospitalar de combate à doença, exercidas por profissionais altamente especializados, utilizando altíssima tecnologia, estão dentro de princípios que os países subdesenvolvidos não podem suportar economicamente.

O estudo comparativo entre a Escandinávia e o Brasil, no que tange a modelos de atenção à saúde bucal, é apropriado desde que o modelo escandinavo tem sido considerado exemplo no Brasil e tem sido recomendado implícita ou explicitamente por associações, escolas e profissionais de odontologia. A recente discussão sobre a adoção de testes bacteriológicos, como contagem de microorganismos para avaliação de risco à cárie, assim como o intercâmbio intenso de profissionais e professores, verificado nas últimas décadas, poderiam ser citados como exemplos de tendência à importação do modelo escandinavo pelo Brasil.

O presente trabalho tem por objetivo analisar as implicações em custos e recursos humanos na trans- 
ferência de um modelo de atenção odontológica praticado em uma sociedade rica e industrializada para um país de Terceiro Mundo, buscando subsidiar estudos mais aprofundados sobre a política nacional de saúde bucal no Brasil.

\section{Modelo Escandinavo - Visão do Sistema Nacional de Saúde Bucal na Suécia}

O Sistema Nacional de Saúde Bucal na Suécia apresenta as seguintes características, de acordo com Barmes $^{1}$ (1975):

- forma de pagamento: indireto, através de seguro social; e direto, onde o paciente paga $60 \%$ do valor do tratamento, desde que este não ultrapasse 3.000 coroas suecas. Se o valor do tratamento for acima desta quantia, o paciente paga $25 \%$ do total;

- tipo de serviço prestado: curativo e preventivo;

- população-alvo: de acordo com a demanda; e também direcionado às necessidades da população infantil e jovem;

- mão-de-obra empregada: privada e pública;

- tipo de recursos humanos utilizados: principalmente profissionais; em menor escala utiliza-se pessoal auxiliar.

Uma característica marcante do modelo sueco é o papel fundamental exercido pelos conselhos regionais. Em número de 26, eles são responsáveis por lei, pelo planejamento e administração dos serviços de saúde dentro de seus limites geográficos, podendo, para isso, impor taxas e impostos aos residentes em suas áreas. Têm autoridade, inclusive, de negociar o estabelecimento de uma nova clínica privada em seu território, assim como estabelecer o número máximo de pacientes que o clínico privado poderá atender em um ano. Assim, tais conselhos regulam fortemente o mercado em relação à saúde na Suécia ${ }^{5}$.

\section{Custos}

Os cuidados com a saúde são responsáveis pelo maior orçamento dentro do setor público sueco 5 . Em 1989, o gasto com saúde representou 9\% do Produto Interno Bruto (PIB) ${ }^{32}$. Os cuidados com saúde bucal consumiram $8,68 \%$ deste orçamento, ou $0,87 \%$ do total do PIB nesse ano $^{32}$.

\section{Quadro Epidemiológico}

A percentagem de crianças livres de cárie no grupo de 3-5 anos de idade foi de $75 \%$ em $1987^{10}$. Para a idade de 7 anos, a percentagem foi semelhante, enquanto para as crianças de 12 anos, o valor foi de $32,3 \%{ }^{10}$.
O CPO-D médio para as crianças de 12 anos de idade foi de 2,6 em 1987 (Tabela 2). Este valor vem decrescendo: em 1985 foi 3,1 e, em 1986, foi de 3, $0^{10}$. Entretanto há diferenças entre as regiões do país: em Estocolmo, o COP-D foi de 4,25, em Kronoberg foi $3,0$, enquanto em Gotemburgo, foi 1,5 (1987) $)^{10}$.

Outros dados relativos às condições de saúde bucal na Suécia estão sumarizados na Tabela 2.

\section{Recursos Humanos}

Em 1990, havia 9.909 cirurgiões-dentistas (CD) exercendo a profissão no País ${ }^{7}$, proporcionando uma relação dentista/habitante de $1 / 860$.

O número de profissionais auxiliares em odontologia, nesse mesmo ano, estava em torno de $15.000^{7}$. Deste total, 1.800 indivíduos eram técnicos de higiene bucal (THD) ${ }^{7}$, o que proporcionava uma relação THD/CD de 0,18/1.

\section{Provisão de Serviços}

O Serviço Nacional de Odontologia na Suécia é responsável pelos cuidados dentais de todos os indivíduos, do nascimento aos 19 anos ${ }^{10}$. Entretanto, 20\% dos adultos são atendidos pelo sistema público, com a expectativa futura de atender $35 \%$ da população adulta do país ${ }^{10}$.

Segundo Bratlhall e col. ${ }^{2}$ (1988), o programa de saúde bucal, colocado à disposição da população na Suécia, é dividido em uma seção básica e uma seção suplementar com o intuito de tornar realizável os procediments mínimos de prevenção por parte dos cirurgiões-dentistas.

O programa básico é normalmente utilizado de forma individual para todos os pacientes, podendo também ser apresentado de forma coletiva. Tem como objetivos previnir o início da doença cárie e da doença periodontal; paralisar o progresso da doença e a recorrência da doença utilizando terapia adequada.

O programa suplementar, quando conveniente, é utilizado em conjunto com o programa básico, objetivando o controle da doença periodontal e da cárie através de medidas específicas.

\section{MATERIAL E MÉTODO}

As sociedades escolhidas para o presente estudo foram a Suécia, país escandinavo, industrializado, rico e com um sistema de bem-estar socializado ${ }^{26}$, e Santa Catarina, Estado do Sul do Brasil, País este classificado como do Terceiro Mundo. O Estado de Santa Catarina foi escolhido por apresentar algumas semelhanças demográficas com 
Tabela 1 - Dados demográficos e socioeconômicos do Brasil, Santa Catarina e Suécia.

\begin{tabular}{lccccccc}
\hline Aspectos & Unidade & \multicolumn{2}{c}{ Brasil } & \multicolumn{2}{c}{ Santa Catarina } & \multicolumn{2}{c}{ Suécia } \\
\hline População & milhões/hab & 150,36 & $(1990)$ & 4,46 & $(1990)$ & 8,33 & $(1990)$ \\
Densidade Demográfica & hab/km2 & 17,7 & $(1990)$ & 38,8 & $(1990)$ & 20,7 & $(1989)$ \\
Taxa cresc. populacional & $\%$ ano & 2,1 & $(1990)$ & 1,7 & $(1990)$ & zero & $(1989)$ \\
Taxa mort. infantil & $\mathrm{n} / 1000$ & 55,0 & $(1990)$ & 26,0 & $(1990)$ & 5,7 & $(1988)$ \\
Taxa analfabetismo & $\%$ & 22,0 & $(1989)$ & 13,5 & $(1990)$ & zero & $(1988)$ \\
Renda per cápita & US\$ & $2.280,00$ & $(1988)$ & $2.950,00$ & $(1988)$ & $19.150,00$ & $(1988)$ \\
\hline
\end{tabular}

Fontes: Governo de Santa Catarina ${ }^{13,14,15}$

Instituto Brasileiro de Geografia e Estatística ${ }^{8,9}$

Government of the Kingdom of Sweden ${ }^{10,11,12}$

World Bank ${ }^{30}$

o país escandinavo escolhido, além de apresentar um dos melhores índices de qualidade de vida, se comparado com outros Estados brasileiros ${ }^{8}$. A Tabela 1 indica alguns dados demográficos e socioeconômicos do Brasil, Santa Catarina e Suécia.

\section{Implicações em Custos}

Para se obter as implicações em custos quando da transferência do modelo de atenção odontológica sueco para Santa Catarina, foi fundamental determinar os recursos humanos necessários para combater os altos níveis de doenças bucais no Estado. Considerou-se aqui a odontologia tradicionalmente exercida no Brasil, por cirurgiõesdentistas e de orientação predominantemente curativa.

Para determinar a necessidade de mão-de-obra, utilizou-se o Programa de Software "Health Through Oral Health" desenvolvido pela Organização Mundial de Saúde e Federação Dentária Internacional em 1989. ${ }^{33}$

Os dados referentes às condições de saúde bucal em Santa Catarina restringiram-se à experiência de cárie aos 12 anos (CPO-D), necessidade de exodontias (\%), necessidade de tratamento periodontal (CPITN) e necessidade de prótese total (\%), de acordo com o levantamento realizado pelo Ministério da Saúde ${ }^{18}$, em 1986. Para o presente estudo considerou-se os dados da região Sul como válidos para o Estado de Santa Catarina. A Tabela 2 sumariza as condições de saúde bucal no Brasil, em Santa Catarina e na Suécia.

Foi também necessário determinar os níveis de demanda ao serviço odontológico em Santa Catarina. Quatro opções foram estabelecidas:
Opção A: de acordo com o levantamento epidemiológico de $1986^{18}$, na região Sul do Brasil, $45 \%$ das crianças de 6 a 12 anos receberam algum tipo de cuidado odontológico naquele ano. A percentagem para a faixa etária de $15-19$ anos foi de $72 \%$ e a média para as faixas etárias de 35-44 anos e 50-59 anos foi de 44\%. Assumiuse que estes números poderiam, de alguma forma, representar o grau de demanda ao serviço odontológico nestas faixas etárias.

$O p c ̧ a \tilde{o}$ B: assumiu-se a demanda de $20 \%$ em todas as faixas etárias estudadas, já que não mais de $20 \%$ da população brasileira pode freqüentar consultórios odontológicos por motivos financeiros ${ }^{23}$.

Opção C: assumiu-se a demanda de $50 \%$ em todas as faixas etárias estudadas.

Opção D: assumiu-se a demanda de $100 \%$ em todas as faixas etárias estudadas.

Para estabelecer as implicações em custos, foi ainda necessário determinar a despesa total por cirurgião-dentista (CD) na Suécia. O valor total foi obtido com a inclusão de renda profissional, gastos com materiais, laboratórios e administração.

\section{Implicações em Recursos Humanos}

As implicações em recursos humanos foram obtidas através dos seguintes estudos comparativos: relação cirurgião-dentista $\mathrm{CD} /$ habitante, relação cirurgião-dentista $\mathrm{CD} /$ técnicos em higiene dental(THD), relação cirurgiãodentista $\mathrm{CD} /$ técnicos em prótese dentária(TPD) e relação cirurgião-dentista $\mathrm{CD} /$ atendente de consultório dentá$\operatorname{rio}(\mathrm{ACD})$.

Tabela 2 - Condições de saúde bucal no Brasil (1986), Santa Catarina (1986) e Suécia (1987).

\begin{tabular}{|c|c|c|c|}
\hline Indicadores & $\begin{array}{r}\text { Brasil } \\
(1986) \\
\end{array}$ & $\begin{array}{c}\text { Santa Catarina } \\
(1986) \\
\end{array}$ & $\begin{array}{l}\text { Suécia } \\
(1987)\end{array}$ \\
\hline CPO-D 12 anos & 6,6 & 6,3 & 2,6 \\
\hline $\begin{array}{l}\% \text { pessoas } \mathrm{CPITN}=4 \\
35 \text { anos de idade }\end{array}$ & 5,1 & 3,8 & zero \\
\hline $\begin{array}{l}\% \text { pessoas CPITN=4 } \\
50 \text { anos de idade }\end{array}$ & 7,4 & 2,0 & 2,0 \\
\hline $\begin{array}{l}\% \text { pessoas necessitando/usando } \\
\text { próteses totais a partir }\end{array}$ & 72,0 & 72,0 & 7,5 (áreas pouco habitadas) \\
\hline dos 50 anos de idade & & & 25,0 (áreas muito habitadas) \\
\hline
\end{tabular}

Fonte: Ministério da Saúde do Brasil ${ }^{18,19}$

Government of the Kingdom of Sweden ${ }^{10,11}$

CPITN = Índice Comunitário de Necessidade de Tratamento Periodontal 


\section{RESULTADOS}

\section{Implicações em Custos}

A Tabela 3 mostra a necessidade de recursos humanos (CD) em Santa Catarina, de acordo com o "Health Through Oral Health" ${ }^{33}$, com as opções de demanda nas faixas etárias estudadas.

O gasto total por CD na Suécia foi determinado após análise dos seguintes dados:

- despesa per capita em serviços odontológicos: US\$ $100.00^{32}$

- número de cirugiões-dentistas trabalhando: 9.9097; - população sueca: 8.330 .000 habitantes ${ }^{32}$.

Multiplicando-se a despesa total per capita em serviços odontológicos pela população daquele país escandinavo obteve-se o gasto total em serviços de atenção à saúde bucal na Suécia: 833 milhões de dólares americanos. Este valor dividido pelo número

Tabela 3 - Necessidade de cirurgiões-dentistas (CD) em Santa Catarina, estimado pelo "Health Through Oral Health"33.

\begin{tabular}{cc}
\hline Opção de Demanda & Proporção CD/n ${ }^{\circ}$ de habitantes \\
\hline A & $1 / 1.528$ \\
B & $1 / 4.000$ \\
C & $1 / 1.1600$ \\
D & $1 / 800$ \\
\hline Legenda: Opçães de percentagens de demanda: \\
Opção A: $45 \%$ da população na faixa etária de 6 a 12 anos; \\
$72 \%$ da população na faixa etária de 15 a 19 anos; \\
$44 \%$ da população nas faixas etárias de 35 a 44 anos e de 50 \\
a 59 anos. \\
A. \\
Opção B: $20 \%$ da população em todas as faixas etárias listadas na opção \\
Opção C: $50 \%$ da população em todas as faixas etárias listadas na opção \\
A. \\
Opção D: $100 \%$ da população em todas as faixas etárias listadas na \\
opção A.
\end{tabular}

de CDs que trabalhavam em 1990, dá o gasto total por CD: 84,06 mil dólares ao ano.

Aplicando-se o gasto total por CD na Suécia, ao número de $\mathrm{CD}$ s determinado nas opções $\mathrm{A}, \mathrm{B}, \mathrm{C}$ e D em Santa Catarina, foram obtidos os valores demonstrados na Tabela 4. A opção D, cuja previsão de demanda por atendimento odontológico é de $100 \%$ da população catarinense, nas faixas etárias estudadas mostrou a necessidade da utilização da quantia astronômica de 468,86 milhões de dólares americanos em gastos com o serviço odontológico no Estado.

\section{Implicações em Recursos Humanos}

Aplicando-se a relação $\mathrm{CD}$ /população existente na Suécia (1CD/860 hab.) a Santa Catarina, o Estado necessitaria 5.186 CDs atuando, isto é, $85 \%$ a mais do que o número existente em 1990 (Tabela 5).

As relações CD/pessoal auxiliar na Suécia eram em 1990, as seguintes:

- 0,88 cirurgião-dentista para cada um atendente de consultório dentário ${ }^{11}$;

- 0,18 cirurgião-dentista para cada um técnico em higiene dental ${ }^{11}$;

- 0,20 cirurgião-dentista para cada um técnico em prótese dentária ${ }^{11}$.

Para que estas relações fossem aplicadas a Santa Catarina, considerando número de CDs existentes em 1990, o Estado deveria formar 3.154 ACDs para juntarem aos 24 ACDs registrados no CRO/SC em $1990^{4}$. Santa Catarina deveria ainda dispor de 506 THDs (somente 1 THD registrado no CRO/SC, em 1990) ${ }^{4}$ e mais 365 TPDs para somarem-se aos 197 registrados no CRO/SC, em $1990^{4}$ (Tabela 5).

Tabela 4 - Gasto total por cirurgião-dentista $(C D)$ na Suécia, aplicado ao número de CDs necessários em Santa Catarina, determinado nas opções A, B, C, e D.

\begin{tabular}{lcc}
\hline Opção & $\mathrm{n}^{\circ}$ CDs necessários & gasto total (milhões de US dólares) \\
\hline $\mathrm{A}(1 \mathrm{CD} / 1.528$ hab.) & 2.919 & 245,37 \\
$\mathrm{~B}(1 \mathrm{CD} / 4.000$ hab.) & 1.115 & 93,72 \\
$\mathrm{C}(1 \mathrm{CD} / 1.600$ hab.) & 2.788 & 234,35 \\
$\mathrm{D}(1 \mathrm{CD} / 800$ hab.) & 5.575 & 468,86 \\
\hline
\end{tabular}

Legenda: A proporção $\mathrm{CD} /$ habitantes foi estimada a partir das opções de demanda nas faixas etárias estudadas. Após esta determinação, aplicou-se o resultado à população de Santa Catarina em todas as faixas etárias, no ano base de 1990.

Tabela 5 - Recursos humanos em Odontologia existentes na Suécia e Santa Catarina em 1990 e necessários em Santa Catarina para atingir as proporções suecas.

\begin{tabular}{lrrr}
\hline Recursos Humanos em Odontologia & $\begin{array}{c}\text { Suécia } \\
(1990)\end{array}$ & $\begin{array}{r}\text { Santa Catarina } \\
(1990)\end{array}$ & $\begin{array}{c}\text { Santa Catarina } \\
\text { (necessários) }\end{array}$ \\
\hline Cirurgiões-Dentistas(CDs) & 9.909 & 2.812 & 3.186 \\
Técnicos de Higiene Dental (THDs) & 1.800 & 01 & 506 \\
Atendentes de Consultório Dentário (ACDs) & 11.200 & 24 & 3.178 \\
Técnicos em Prótese Dental (TPDs) & 2.000 & 197 & 562 \\
\hline
\end{tabular}

Fonte: Fédération Dentaire Internationale

Conselho Regional de Odontologia de Santa Catarina 


\section{DISCUSSÃO}

Para a opção A, levou-se em consideração uma demanda por serviços odontológicos semelhante àquelas levantadas pelo estudo do Ministério da Saúde em $1986^{18}$. Sabe-se, no entanto, as limitações que estes dados apresentam, uma vez que derivaram da resposta dos pesquisados ao serem indagados se haviam procurado atendimento odontológico nos últimos 12 meses. Mesmo entendendo-se que este não seja um bom indicador de demanda, justifica-se sua utilização pela carência de informações a este respeito. Assim sendo, para estes índices de demanda e ainda considerando-se os níveis de saúde bucal encontrados em Santa Catarina, necessitar-se-ia 2.919 CDs atuando no Estado. Aplicando-se o total de gastos por CD na Suécia, o Estado brasileiro estudado deveria dispor de 245,37 milhões de dólares americanos/ano para aplicar no serviço público odontológico. Este valor representaria quatro vezes o orçamento total do Estado ${ }^{13}$ (1990) destinado à saúde.

Para a opção B de demanda, o total de gastos representaria 1,5 vezes o orçamento total destinado à saúde $^{13}$ (1990). Para a opção C de demanda, a situação seria similar à opção $\mathrm{A}$. Para a opção $\mathrm{D}$, onde $100 \%$ da população das faixas etárias de estudo demandasse aos serviços odontológicos, o Estado necessitaria 8 vezes todo o orçamento de saúde de $1990^{13}$. Mesmo que fossem dividos os valores obtidos nas 4 opões do estudo, pela metade, o que seria justificável pela diferença de população entre Santa Catarina e Suécia, os valores ainda assim seriam irrealistas.

Nas opções A e C, o número de CDs necessários é muito similar ao que Santa Catarina dispunha em 1990, e que na opção B, o número de CDs é menor do que a metade do número que estava atuando no mesmo ano (Tabela 4).

A aplicação do modelo sueco de atenção odontológica em Santa Catarina seria inviável, não podendo o Estado suportá-lo devido a alta necessidade de alocação de recursos financeiros.

Com relação às implicações em recursos humanos, a proporção $\mathrm{CD} /$ habitantes da Suécia, aplicada a Santa Catarina, exigiria mais $85 \%$ de CDs atuando no Estado. De acordo com Werner ${ }^{29}$ (1987), o número de CDs que irão trabalhar no Brasil no ano 2010 poderá atingir 208.896, se for levado em consideração a percentagem de $5 \%$ de profissionais que deixam a profissão anualmente. Em 1990, 2,64\% dos CDs brasileiros trabalhavam em Santa Catarina ${ }^{4}$. Se esta percentagem se mantiver, haverá no ano 2010, 5.500 CDs atuando no Estado, o que proporcionaria uma relação de um $\mathrm{CD} / 1.236$ habitantes, já que a estimativa para a população catarinense para o ano 2010 é de 6,8 milhões de habitantes ${ }^{14}$. Esta relação ainda estaria longe da apresentada pela Suécia. Entretanto, como o processo de abertura de novas escolas de odontologia no Brasil está fora de controle ${ }^{29}$, ou estava até pouco tempo, torna-se impossível qualquer previsão.

No tocante ao pessoal auxiliar, a situação ainda é mais difícil. Há poucas escolas formadoras no Estado de Santa Catarina e pouca evidência de que os setores público e privado desejem estimular a formação de auxiliares em odontologia.

Como foi citado anteriormente, Santa Catarina apresenta indicadores socioeconômicos razoavelmente melhores do que a média nacional. Embora os resultados do presente estudo reportem-se apenas a dados deste Estado, inevitável se faz o questionamento acerca da utilização do modelo escandinavo de atenção odontológica em outras regiões ou Estados brasileiros com indicadores socioeconômicos menos favoráveis que Santa Catarina. Pode-se inferir, à luz do presente estudo, que os resultados seriam irrealistas.

De acordo com os presentes achados, a adoção dos princípios de alta provisão de recursos financeiros e humanos, característico do modelo sueco e escandinavo, por Santa Catarina e pelo Brasil, estaria totalmente fora da realidade. Não se poderia, portanto, considerá-los exemplo para o Brasil. As realidades geográficas, demográficas, sociais, econômicas, culturais e políticas são divergentes. Necessário se faz a busca de caminhos próprios, coerentes com a realidade do País, não negando os avanços científicos advindos de sociedades que já atingiram um patamar de desenvolvimento maior que o do Brasil. Também não se deve negar os avanços obtidos por vários municípios brasileiros, principalmente no que tange a êxitos epidemiológicos em relação à cárie dental. Essas localidades buscaram alternativas diferentes, de acordo com suas realidades loco-regionais. Dentre essas destacam-se a água de abastecimento fluoretada e sua efetiva vigilância, escovação supervisionada com creme dental fluoretado em escolas, entre outras. Este êxito epidemiológico pode ser constatado na Tabela 6.

O modelo de atenção em saúde bucal para o Brasil não pode, portanto, ser baseado em atenção individual que dependa de profissionais e equipamentos odontológicos, o que ocasionaria um custo extremamente alto, mas sim em políticas de promoção de saúde que possam ser de alcance da maioria da população. Como exemplos pode-se citar a fluoretação 
Tabela 6 - CPO-D aos 12 anos de idade em alguns municípios brasileiros.

\begin{tabular}{lllc}
\hline \multicolumn{1}{c}{ Município } & \multicolumn{1}{c}{ Autores } & CPO-D \\
\hline São José dos Campos (SP) & Rosa e col. $^{27}$ & 1992 & 3,96 \\
Ipatinga (MG) & Peres $^{21}$ & 1995 & 2,80 \\
Araucária (PR) & Rocha \& Fonseca $^{25}$ & 1994 & 3,49 \\
Jaraguá do Sul (SC) & Clavera $^{3}$ & 1994 & 4,47 \\
Santos (SP) & Pref. Mun. Santos $^{24}$ & 1994 & 1.73 \\
Balneário Camboriú (SC) & Lunardelli $^{17}$ & 1994 & 4,12 \\
Florianópolis (SC) & Peres e col. & 1995 & 2,71 \\
Blumenau (SC) & Traebert e col. ${ }^{28}$ & 1995 & 2,87 \\
\hline
\end{tabular}

das águas de abastecimento e sua vigilância, ou o tratamento das águas em áreas menos favorecidas, o acesso universal ao creme dental fluoretado e aos meios de higiene bucal, uma política mais adequada de alimentos visando reduzir o consumo de açúcar e estimular a utilização, principalmente em nível industrial, dos substitutos da sacarose, ampla formação de pessoal auxiliar em odontologia, com o intuito de fortalecer a educação em saúde e proporcionar o aumento das equipes de trabalho destinadas ao atendimento da população, e, finalmente, uma melhor distribuição de renda, melhorando as condições de vida e ampliando o acesso aos meios de prevenção às doenças bucais mais prevalentes. $\mathrm{O}$ modelo deve basear-se na promoção de saúde, incluindo prevenção e educação, que não necessariamente depende

\section{REFERÊNCIAS BIBLIOGRÁFICAS}

1. BARMES, D.E. Current systems of delivery of oral health services. Int. Dent. J., 25: 20-5, 1975.

2. BRATLHALL, D. et. al. O Programa Sueco de Saúde Oral para Adultos. RBO, 6: 2-10, 1988.

3. CLAVERA, W.V. Vigilância epidemiológica no planejamento e avaliação dos serviços municipais de saúde. Divulg. Saúde Debate, 10: 72, 1995.

4. CONSELHO REGIONAL DE ODONTOLOGIA DE SANTA CATARINA. Ofício nº 273/91. Florianópolis, 1991.

5. ENTOVEN, C.A. The Swedish dental care system. Stockholm, Sweden Board of Statistics, 1985.

6. EVANG, K. Health service, society and medicine. London, Oxford University Press, 1975.

7.FÉDÉRATION DENTAIRE INTERNATIONALE. Basic facts 1990 - Dentistry around the world. London, Jones and Palmer, 1990.

8. FUNDAÇÃO IBGE. Informações básicas 1987. Rio de Janeiro, 1987. de recursos humanos humanos odontológicos e de alto custo, e não somente no tratamento da doença, embora esta, quando já instalada, deva ter seu tratamento propiciado através de um amplo acesso aos serviços. Estes devem estar adequados quanto à tecnologia, que deve ser apropriada, e priorizações, de acordo com o quadro epidemiológico vigente.

\section{AGRADECIMENTOS}

Aos professores Maria do Carmo Matias Freire, do Departamento de Odontologia Social da Universidade Federal de Goiás (UFG) e Marco Aurélio de Anselmo Peres, do Departamento de Saúde Pública da Universidade Federal de Santa Catarina (UFSC), pela revisão e sugestões.

9. FUNDAÇÃO IBGE. Estimativa da população residente. Rio de Janeiro, 1990

10. GOVERNMENT OF THE KINGDOM OF SWEDEN. Oral health and dental care in Sweden. Stockholm, Sweden Board of Statistics, 1988.

11. GOVERNMENT OF THE KINGDOM OF SWEDEN. Statistical abstract of Sweden 1991. Stockholm, Statistics Sweden, 1991.

12. GOVERNMENT OF THE KINGDOM OF SWEDEN Useful information on Social Security. Stockholm, Forsakringskasseforbundet, 1991.

13. GOVERNO DO ESTADO DE SANTA CATARINA. Desempenho geral da economia catarinense. Florianópolis, Imprensa Oficial, 1989.

14. GOVERNO DO ESTADO DE SANTA CATARINA. Santa Catarina, estudo da evolução populacional 1970-2010. Florianópolis, Imprensa Oficial, 1989.

15. GOVERNO DO ESTADO DE SANTA CATARINA. Séries estatísticas de Santa Catarina. Florianópolis, Imprensa Oficial, 1990. 
16. LIND, P.O. \& MOLLER, J.J. New trends in the delivery of oral health care. Efurt, WHO, 1983.

17. LUNARDELLI, S.E. Prevalência de cárie dental no município de Balneário Camboriú SC, 1994: relatório preliminar. Balneário Camboriú, 1994.

18. MINISTÉRIO DA SAÚDE DO BRASIL. Levantamento epidemiológico em saúde bucal. Brasil, zona urbana, 1986. Brasília, Centro de Documentação do Ministério da Saúde, 1988.

19. MINISTÉRIO DA SAÚDE DO BRASIL. Perfil da odontologia brasileira. Brasília, 1988. [DNSB-Documento técnico 01/88].

20. NAVARRO, V. The underdevelopment of health and health of underdevelopment: an analyses of the distribution of human resources in Latin America. Int. J. Health Serv., 4:5-27, 1974.

21. PERES, M.A. A saúde bucal em um sistema local de saúde: estudo de caso - Município de Itapinga MG, Brasil, 19891994. São Paulo, 1995. [Dissertação de Mestrado Faculdade de Saúde Pública da USP].

22. PERES, M.A. et al. Prevalência de cárie dentária na população de 3 a 12 anos de idade no Município de Florianópolis, SC, Brasil, 1995: relatório preliminar. Florianópolis, 1995.

23. PINTO, V.G. Saúde bucal no Brasil. Rev. Saúde Pública, 17:316-27, 1983.

24. PREFEITURA MUNICIPAL DE SANTOS. Levantamento epidemiológico em saúde bucal dos escolares do Município de Santos, 1995: relatório preliminar. Santos, 1995.
25. ROCHA, A.C. \& FONSECA, R.S. Censo epidemiológico de cárie aos 12 anos em Araucária PR. Divulg. Saúde Debate, 10:70, 1995.

26. ROEMER, M. Health care systems in world perspectives. Los Angeles, Health Administration Press, 1976.

27. ROSA. A.G. et al. Programas de reorientação do atendimento odontológico escolar com ênfase na prevenção. RGO, 40:110-4, 1992.

28. TRAEBERT, J.L. et al. Prevalência e cárie em escolares do Município de Blumenau SC, 1995: relatório preliminar. Blumenau, 1995.

29. WERNER, C.W. The decision-making process in the opening of new dental schools in Brazil. London, 1987 [Tese de Mestrado - University of London].

30. WORLD BANK. Social indicators of development. London, University Press, 1989.

31. WORLD HEALTH ORGANIZATION. Oral health care systems - an international collaborative study. London, Quintessence Publishing Co., 1985.

32. WORLD HEALTH ORGANIZATION. Financing of dental care in Europe. Copenhaguen, 1990.

33. WORLD HEALTH ORGANIZATION \& FÉDÉRATION DENTAIRE INTERNATIONALE. Health through oral health - guidelines for planning and monitoring for oral health care. London, Quintessence Publishing Co., 1989. 